\title{
An embedding theorem for Sobolev type functions with gradients in a Lorentz space
}

by

\author{
Alireza Ranjbar-Motlagh (Tehran)
}

\begin{abstract}
The purpose of this paper is to prove an embedding theorem for Sobolev type functions whose gradients are in a Lorentz space, in the framework of abstract metricmeasure spaces. We then apply this theorem to prove absolute continuity and differentiability of such functions.
\end{abstract}

Introduction. In this article, we extend Morrey's embedding theorem (see for instance [GT, Thm. 7.17]) to Sobolev type functions whose generalized gradients are in a Lorentz space, when the underlying space is a metric-measure space. In fact, Morrey's theorem states that for any function $f$ in the Sobolev space $W^{1, p}\left(\mathbb{R}^{n}\right)$, the following inequality is satisfied:

$$
\operatorname{esssup}_{x, y \in B(a, R)}|f(x)-f(y)| \leq C R^{1-n / p}\|\nabla f\|_{L^{p}},
$$

whenever $p>n$, for some constant $C$ which depends on $n$ and $p$. We state and prove an inequality, similar to (1), controlling the value of $\operatorname{ess} \sup _{x, y \in B(a, R)}|f(x)-f(y)|$ by the Lorentz norm of the (generalized) gradient of $f$, for a real-valued function $f$ whose domain is a metric-measure space (see Theorem 2.1). We use the definition of Sobolev spaces as in $[\mathrm{H}]$, in order to define the (generalized) gradient for functions over abstract spaces. Then we study continuity and differentiability of such functions. Our results sharpen those of $[\mathrm{MS}]$ for weighted Sobolev spaces and extend the main results of $[\mathrm{KKM}]$ to metric-measure spaces. See also $[\mathrm{AC}],[\mathrm{DS}],[\mathrm{S}],[\mathrm{Cc}],[\mathrm{CL}]$ and $[\mathrm{Ca}]$ for other work on this subject.

1. Preliminaries. First, we recall some basic definitions relating to metric-measure spaces, that is, metric spaces $(X, d)$ with a Radon (outer)

2000 Mathematics Subject Classification: Primary 26D10, 46E30; Secondary 46E35, 26B30. Key words and phrases: Lorentz spaces, Sobolev functions, differentiability, absolutely continuous functions, Orlicz spaces. 
measure $\mu$. We denote by $B(x, r)$ the open ball of radius $r>0$ with center at $x$ in a metric space $(X, d)$. For the basic concepts of metric-measure spaces, see for instance $[\mathrm{He}]$ and the references therein.

Definition 1.1. Let $(X, d, \mu)$ be a metric-measure space and let $\Omega$ be a (measurable) subset of $X$. Then $\Omega$ is also a metric-measure space with the induced metric and measure. We say that $\Omega$ is Bishop-Gromov regular of dimension $n>0$ if

$$
\frac{\mu(B(x, s) \cap \Omega)}{s^{n}} \leq C_{0} \frac{\mu(B(y, r) \cap \Omega)}{r^{n}}
$$

for all $0<r<2 s, x, y \in \Omega$ and $d(x, y) \leq s$, where $C_{0} \geq 1$ is a constant.

We say that $\Omega$ is doubling if there is a constant $C^{\prime}>0$ such that

$$
\mu(B(x, 2 r) \cap \Omega) \leq C^{\prime} \mu(B(x, r) \cap \Omega)
$$

for all $x \in \Omega$ and $r>0$.

It is easy to see that if $\Omega$ is doubling, then it is Bishop-Gromov regular of dimension $n$ for some $n>0$. Also, if $\Omega$ is doubling and $\mu$ is not identically zero on $\Omega$, then $\mu(B(x, r) \cap \Omega)>0$ for all $x \in \Omega$ and $r>0$.

Now, we recall the definition of Lorentz spaces. For their basic properties, see for instance $[\mathrm{SW}],[\mathrm{BS}]$ and $[\mathrm{KK}]$; see also $[\mathrm{CRS}]$ and the references therein.

Definition 1.2. Let $(X, d, \mu)$ be a metric-measure space and let $\Omega$ be a (measurable) subset of $X$. Let $u: X \rightarrow \mathbb{R}$ be a measurable function and let $m$ be a positive number. We say that $u$ belongs to the Lorentz space $\mathcal{L}^{m, 1}(\Omega)$ if

$$
\|u\|_{\mathcal{L}^{m, 1}(\Omega)}:=\int_{0}^{\infty} \mu(\{z \in \Omega:|u(z)|>t\})^{1 / m} d t<\infty .
$$

Next, we state the following simple fact about connected doubling metricmeasure spaces (cf. Remark 2.2).

Proposition 1.3. Let $(X, d, \mu)$ be a metric-measure space and assume that the ball $B(a, R)$ is a connected doubling subset of $X$. Suppose that $\mu$ is not identically zero on $B(a, R)$ and there exists $\left.R^{-} \in\right] 2 R / 3, R[$ such that $B\left(a, R^{-}\right) \neq B(a, R)$. Then

$$
0<\mu(B(x, r) \cap B(a, R))<\mu(B(x, r+t) \cap B(a, R))<\infty
$$

for all $x \in B(a, R), r \in] 0, R / 4]$ and $t>0$.

Proof. Since $B(a, R)$ is doubling and $\mu$ is not identically zero on $B(a, R)$, we know that $\mu(B(y, s) \cap B(a, R))>0$ for all $y \in B(a, R)$ and $s>0$. First, assume $R / 4<d(x, a)<R$. Since the distance function is continuous and 
$B(a, R)$ is connected, there exist $z \in X$ and $s>0$ such that

$$
B(z, s) \subset[B(x, r+t) \backslash B(x, r)] \cap B(a, R)
$$

for any $r \in] 0, R / 4]$ and $0<t<d(x, a)-R / 4$. So, the condition (2) holds.

Also, when $d(x, a) \leq R / 4$, by a similar method (and using the condition $\left.B\left(a, R^{-}\right) \neq B(a, R)\right)$, we can obtain (2).

Now, we recall the concept of geodesic spaces. Let $(X, d)$ be a metric space. We say that $X$ is a geodesic space if for any $x, y \in X$, there exists a geodesic $\gamma_{x, y}$ between $x$ and $y$, i.e. there is an isometry $\gamma_{x, y}:[0, d(x, y)] \rightarrow X$ such that $\gamma(0)=x$ and $\gamma(d(x, y))=y$.

Proposition 1.4. Let $(X, d, \mu)$ be a metric-measure space and let $B(a, R)$ be a ball in $X$. Suppose that $X$ is geodesic and Bishop-Gromov regular of dimension n, i.e.

$$
\frac{\mu(B(x, s))}{s^{n}} \leq C_{0} \frac{\mu(B(y, r))}{r^{n}}
$$

for all $0<r<2 s, x, y \in X$ and $d(x, y) \leq s$, where $C_{0} \geq 1$ is a constant. Then $B(a, R)$ is Bishop-Gromov regular of dimension $n$, more precisely,

$$
\frac{\mu(B(x, s) \cap B(a, R))}{s^{n}} \leq C \frac{\mu(B(y, r) \cap B(a, R))}{r^{n}}
$$

for all $0<r<2 s, x, y \in B(a, R)$ and $d(x, y) \leq s$, where $C$ is a constant which depends on $C_{0}$ and $n$, but is independent of $a$ and $R$.

Proof. Pick $x, y \in B(a, R)$ and $r, s \in \mathbb{R}$ with $0<r<2 s$ and $d(x, y) \leq s$. It is clear that if $B(y, r) \subset B(a, R)$, we immediately obtain the desired result. Also, without loss of generality, we may assume $r \leq R / 2$. Since $X$ is geodesic, by considering a geodesic $\gamma_{a, y}$ between $a$ and $y$, we see that there exists $z \in B(a, R)$ such that

$$
B(z, r / 2) \subset B(y, r) \cap B(a, R) .
$$

Moreover, since $X$ is doubling, we know that

$$
\mu(B(y, r)) \leq C \mu(B(z, r / 2)),
$$

where $C$ is a constant which depends on $C_{0}$ and $n$. This completes the proof of the proposition.

2. Embedding theorem. In this section, we state and prove an embedding theorem for Sobolev type functions with gradients in the Lorentz space $\mathcal{L}^{n, 1}(X)$. We use a Lipschitz type characterization of Sobolev spaces (see $[\mathrm{H}]$ ) to define the (generalized) gradient for Sobolev type functions over metric-measure spaces.

Theorem 2.1. Let $(X, d, \mu)$ be a metric-measure space and assume that the ball $B(a, R)$ is Bishop-Gromov regular of dimension $n$. Suppose that 
$B(a, R)$ is connected, $\mu$ is not identically zero on $B(a, R)$ and there exists $\left.R^{-} \in\right] 2 R / 3, R\left[\right.$ such that $B\left(a, R^{-}\right) \neq B(a, R)$. Suppose that $f: X \rightarrow \mathbb{R}$ is a measurable function satisfying

$$
|f(x)-f(y)| \leq d(x, y)[g(x)+g(y)]
$$

for a.e. $x, y \in X$, where $g$ is a non-negative function in the Lorentz space $\mathcal{L}^{n, 1}(X)$. Then

$$
|f(x)-f(y)| \leq 1000\left(\frac{C_{0} R^{n}}{\mu(B(a, R))}\right)^{1 / n}\|g\|_{\mathcal{L}^{n, 1}(B(a, R))}
$$

for a.e. $x, y \in B(a, R)$, where the constant $C_{0}$ is as in Definition 1.1 (with $\Omega=B(a, R))$.

Proof. Set $\Omega:=B(a, R)$. There is a set $E \subset X$ of measure zero such that

$$
|f(z)-f(w)| \leq d(z, w)[g(z)+g(w)]
$$

for all $z, w \in X \backslash E$. For $i \in \mathbb{Z}$, define

$$
\begin{gathered}
F_{i}:=\left\{z \in \Omega: g(z) \leq 2^{i}\right\} \backslash E, \\
r_{i}:=\min \left\{2 R,\left(\mu\left(\Omega \backslash F_{i}\right) \frac{C_{0} R^{n}}{\mu(\Omega)}\right)^{1 / n}\right\}, \quad s_{i}:= \begin{cases}r_{i} & \text { if } r_{i} \leq R / 4, \\
2 R & \text { if } r_{i}>R / 4 .\end{cases}
\end{gathered}
$$

First, we show that

$$
\mu\left(\Omega \backslash F_{i}\right) \leq \mu\left(B\left(x, r_{i}\right) \cap \Omega\right)
$$

for all $i \in \mathbb{Z}$ and $x \in \Omega$. If $r_{i}=2 R$ or $r_{i}=0$, then (4) is obvious; otherwise, $0<r_{i}<2 R$, and since $\Omega$ is Bishop-Gromov regular, we have

$$
\frac{\mu(\Omega)}{R^{n}} \leq C_{0} \frac{\mu\left(B\left(x, r_{i}\right) \cap \Omega\right)}{r_{i}^{n}}
$$

for all $i \in \mathbb{Z}$ and $x \in \Omega$. This implies (4).

Next, we show that for any $x_{k+1} \in F_{k+1}$, whenever $F_{k} \neq \emptyset$ there exists $x_{k} \in F_{k}$ such that $d\left(x_{k+1}, x_{k}\right)<s_{k}^{+}$for any $s_{k}^{+}$such that $\mu\left(B\left(x_{k+1}, s_{k}\right) \cap \Omega\right)$ $<\mu\left(B\left(x_{k+1}, s_{k}^{+}\right) \cap \Omega\right)$; when $s_{k}=2 R$ define $s_{k}^{+}:=s_{k}=2 R$. This is obvious if $r_{k}>R / 4$, so suppose that $r_{k} \leq R / 4$. By contradiction, suppose that $B\left(x_{k+1}, s_{k}^{+}\right) \cap F_{k}=\emptyset$. Then $B\left(x_{k+1}, s_{k}^{+}\right) \cap \Omega \subset \Omega \backslash F_{k}$, and therefore $\mu\left(B\left(x_{k+1}, s_{k}^{+}\right) \cap \Omega\right) \leq \mu\left(\Omega \backslash F_{k}\right)$. Also, by (4), we obtain $\mu\left(B\left(x_{k+1}, s_{k}^{+}\right) \cap \Omega\right) \leq \mu\left(\Omega \backslash F_{k}\right) \leq \mu\left(B\left(x_{k+1}, s_{k}\right) \cap \Omega\right)<\mu\left(B\left(x_{k+1}, s_{k}^{+}\right) \cap \Omega\right)$, a contradiction.

Now, we prove (3). Suppose that $x, y \in \Omega \backslash E$. There are $k_{x}, k_{y} \in \mathbb{Z}$ such that $g(x) \leq 2^{k_{x}}$ and $g(y) \leq 2^{k_{y}}$. Define $l:=\inf \left\{i: F_{i} \neq \emptyset\right\} \in \mathbb{Z} \cup\{-\infty\}$. 
Then there are two sequences $\left\{x_{i}\right\}$ and $\left\{y_{j}\right\}$ such that

$$
\left\{\begin{array}{l}
x_{k_{x}}:=x \\
x_{i} \in F_{i} \\
d\left(x_{i}, x_{i-1}\right)<s_{i-1}^{+} \quad \text { for } k_{x} \geq i>l
\end{array}\right.
$$

and

$$
\left\{\begin{array}{l}
y_{k_{y}}:=y \\
y_{j} \in F_{j} \\
d\left(y_{j}, y_{j-1}\right)<s_{j-1}^{+} \quad \text { for } k_{y} \geq j>l
\end{array}\right.
$$

Also, we have

$$
|f(z)-f(w)| \leq d(z, w)[g(z)+g(w)] \leq d(z, w)\left[2^{i}+2^{i}\right] \leq 2^{i+1} d(z, w)
$$

for all $z, w \in F_{i}$. Therefore, we obtain

$$
\left|f\left(x_{i}\right)-f\left(x_{i-1}\right)\right| \leq 2^{i+1} s_{i-1}^{+}, \quad\left|f\left(y_{j}\right)-f\left(y_{j-1}\right)\right| \leq 2^{j+1} s_{j-1}^{+},
$$

for all $k_{x} \geq i>l$ and $k_{y} \geq j>l$. Without loss of generality, we may assume that $k_{x} \geq k_{y}$. Then, for any integer $k_{y}>m>l$, we get

$$
\begin{aligned}
|f(x)-f(y)| \leq & \sum_{k_{x} \geq i>m}\left|f\left(x_{i}\right)-f\left(x_{i-1}\right)\right|+\left|f\left(x_{m}\right)-f(y)\right| \\
\leq & \sum_{k_{x} \geq i>m}\left|f\left(x_{i}\right)-f\left(x_{i-1}\right)\right|+\left|f\left(x_{m}\right)-f\left(y_{m}\right)\right|+\left|f\left(y_{m}\right)-f(y)\right| \\
\leq & \sum_{k_{x} \geq i>m}\left|f\left(x_{i}\right)-f\left(x_{i-1}\right)\right|+\left|f\left(x_{m}\right)-f\left(y_{m}\right)\right| \\
& +\sum_{k_{y} \geq j>m}\left|f\left(y_{j}\right)-f\left(y_{j-1}\right)\right| \\
\leq & \sum_{k_{x} \geq i>m} 2^{i+1} s_{i-1}^{+}+2^{m+2} R+\sum_{k_{y} \geq j>m} 2^{j+1} s_{j-1}^{+} \\
\leq & 2^{m+2} R+2 \sum_{k_{x} \geq i>m} 2^{i+1} s_{i-1}^{+} \\
\leq & \left(\frac{C_{0} R^{n}}{\mu(\Omega)}\right)^{1 / n}\left(2^{m+2} \mu(\Omega)^{1 / n}\right)+2 \sum_{k_{x} \geq i>m} 2^{i+1} s_{i-1}^{+} .
\end{aligned}
$$

By Proposition 1.3, we can choose $s_{i-1}^{+}$to be any number greater than $s_{i-1}$, so

$$
|f(x)-f(y)| \leq\left(\frac{C_{0} R^{n}}{\mu(\Omega)}\right)^{1 / n}\left(2^{m+2} \mu(\Omega)^{1 / n}\right)+4 \sum_{\max \left\{k_{x}, k_{y}\right\} \geq i>m} 2^{i+1} s_{i-1}
$$

for all $x, y \in \Omega \backslash E$ and $\min \left\{k_{x}, k_{y}\right\}>m>l$. 
On the other hand, by definition, we have

$$
\sum_{i \in \mathbb{Z}} 2^{i-1} \mu\left(\Omega \backslash F_{i}\right)^{1 / n} \leq\|g\|_{\mathcal{L}^{n, 1}(\Omega)} .
$$

Then, if $l=-\infty$, by letting $m \rightarrow-\infty$ in (5), we obtain

$$
|f(x)-f(y)| \leq 0+600\left(\frac{C_{0} R^{n}}{\mu(\Omega)}\right)^{1 / n}\|g\|_{\mathcal{L}^{n, 1}(\Omega)}
$$

for all $x, y \in \Omega \backslash E$. Also, if $l \neq-\infty$, we know that

$$
2^{l-1} \mu(\Omega)^{1 / n} \leq \int_{0}^{2^{l-1}} \mu(\{z \in \Omega: g(z)>t\})^{1 / n} d t \leq\|g\|_{\mathcal{L}^{n, 1}(\Omega)} ;
$$

then, by choosing $m=l+1$ and (5), we obtain

$$
|f(x)-f(y)| \leq 1000\left(\frac{C_{0} R^{n}}{\mu(\Omega)}\right)^{1 / n}\|g\|_{\mathcal{L}^{n, 1}(\Omega)}
$$

for all $x, y \in \Omega \backslash E$. This completes the proof of the theorem.

REMARK 2.2. In Theorem 2.1, we can replace the connectedness assumption and the condition $B\left(a, R^{-}\right) \neq B(a, R)$ by the following (weaker) assumption:

- There exists a constant $K>1$ such that, for any $x \in B(a, R)$ and $r \in] 0, R / 4]$, we have $\mu(B(x, r) \cap B(a, R))<\mu(B(x, K r) \cap B(a, R))$.

3. Applications. In this section, we apply Theorem 2.1 to study the continuity and differentiability of Sobolev type functions. First, we recall some definitions (see [M1] and [KKM]).

Definition 3.1. Let $(X, d, \mu)$ be metric-measure space and let $f$ be a real-valued function on $X$. We say that $f$ is essentially $m$-absolutely continuous, for some $m>0$, if for any $\varepsilon>0$, there is $\delta>0$ such that for any family $\left\{B_{i}\right\}$ of pairwise disjoint balls in $X$, we have

$$
\sum_{i} \operatorname{esssup}_{x, y \in B_{i}}|f(x)-f(y)|^{m}<\varepsilon \quad \text { whenever } \quad \sum_{i} \mu\left(B_{i}\right)<\delta .
$$

Also, the essential m-variation of $f$ is defined by

$$
\mathcal{V}_{m}(f):=\sup _{\left\{B_{i}\right\}}\left\{\sum_{i} \operatorname{esssup}_{x, y \in B_{i}}|f(x)-f(y)|^{m}\right\},
$$

where the supremum is taken over all pairwise disjoint families $\left\{B_{i}\right\}$ of balls in $X$. 
TheOREM 3.2. Let the notations and assumptions be as in Theorem 2.1. Moreover, assume that $X$ is Bishop-Gromov regular of dimension $n$ and geodesic. Then

(i) For a.e. $a \in X$, we have

$$
\limsup _{s \rightarrow 0} \operatorname{esssup}_{x, y \in B(a, s)} \frac{|f(x)-f(y)|}{s}<\infty .
$$

(ii) There exists a constant $C$ which depends on $C_{0}$ and $n$ (as in Definition 1.1 with $\Omega=X$ ) such that

$$
\mathcal{V}_{n}(f) \leq C\left(\sup _{z, s} \frac{s^{n}}{\mu(B(z, s))}\right)\|g\|_{\mathcal{L}^{n, 1}(X)}^{n} .
$$

(iii) In addition, if $\sup _{z, s} s^{n} / \mu(B(z, s))<\infty$, then $f$ is essentially $n$ absolutely continuous.

Proof. First, we assume $n>1$. We have (cf. [M2, Thm. 2.1]) $\|g\|_{\mathcal{L}^{n, 1}(B(a, R))}$

$$
=\left(\int_{0}^{\infty} \Phi^{\prime}(t)^{1 /(1-n)} d t\right)^{(n-1) / n}\left(\int_{0}^{\infty} \Phi^{\prime}(t) \mu(\{z \in B(a, R): g(z)>t\}) d t\right)^{1 / n},
$$

where $\Phi(t):=\int_{0}^{t} \mu(\{z \in B(a, R): g(z)>s\})^{(1-n) / n} d s$. Then we obtain

$$
\|g\|_{\mathcal{L}^{n, 1}(B(a, R))} \leq L\|g\|_{\mathcal{L}^{n, 1}(B(a, R))}^{1 / n}=L\left(\int_{B(a, R)} \Phi \circ g d \mu\right)^{1 / n}<\infty,
$$

where $L:=\|g\|_{\mathcal{L}^{n, 1}(X)}^{(n-1) / n}<\infty$. Also, it is easy to see that (6) is meaningful and valid when $n=1$ (with $L=1$ and $\Phi(t) \equiv t$ ).

Then, by Proposition 1.4, (3) and (6), we have

$$
\begin{aligned}
\operatorname{esssup}_{x, y \in B(a, s)} \frac{|f(x)-f(y)|}{s} & \leq \frac{1000}{s}\left(\frac{C s^{n}}{\mu(B(a, s))}\right)^{1 / n}\|g\|_{\mathcal{L}^{n, 1}(B(a, s))} \\
& \leq L C\left(\frac{1}{\mu(B(a, s))} \int_{B(a, s)} \Phi \circ g d \mu\right)^{1 / n},
\end{aligned}
$$

where $C$ is a constant which depends on $C_{0}$ and $n$ (associated with $X$ ). Now, Lebesgue's differentiation theorem (for doubling spaces, see for instance [He, Thm. 1.8]) implies (i). On the other hand, assume that $\left\{B\left(a_{i}, s_{i}\right)\right\}$ is any family of pairwise disjoint balls in $X$. Again, by Proposition 1.4, (2.1) and (3.1), we get 


$$
\begin{aligned}
\sum_{i} \operatorname{essiup}_{x, y \in B\left(a_{i}, s_{i}\right)}|f(x)-f(y)|^{n} & \leq L^{n} C^{n} \sum_{i} \frac{s_{i}^{n}}{\mu\left(B\left(a_{i}, s_{i}\right)\right)} \int_{B\left(a_{i}, s_{i}\right)} \Phi \circ g d \mu \\
& \leq L^{n} C^{n}\left(\sup _{z, s} \frac{s^{n}}{\mu(B(z, s))}\right) \sum_{i} \int_{B\left(a_{i}, s_{i}\right)} \Phi \circ g d \mu \\
& =L^{n} C^{n}\left(\sup _{z, s} \frac{s^{n}}{\mu(B(z, s))}\right) \int_{\cup_{i} B\left(a_{i}, s_{i}\right)} \Phi \circ g d \mu .
\end{aligned}
$$

This completes the proof of theorem.

REMARK 3.3. If we replace "ess sup" by "sup" in Definition 3.1 and Theorem 3.2(iii), then the conclusion of Theorem 3.2(iii) remains valid after changing $f$ on a set of measure zero.

Now, by the implications of the Poincaré inequality [HK, Thms. 3.2 and 3.4], properties of the Hardy-Littlewood maximal function [He, Thm. 2.2] (see also [KK, Thm. 1.2.1]), Marcinkiewicz's interpolation theorem for Lorentz spaces [BS, Ch. 4, Thm. 4.13, Rem. 4.15], characterization of the Lorentz space $\mathcal{L}^{n, 1}(X)$ by Orlicz spaces [Cc] (see also [CL], [KKM] and [AC]) and properties of $A_{p}$-weights [KK, Thm. 5.3.1], we are able to recover and sharpen the results of $[\mathrm{MS}]$ for $A_{p}$-weighted Sobolev functions. Moreover, Theorem 3.2 extends the main results of [KKM] and [AC, Thm. 1.1] (see also $[\mathrm{Cc}]$ and $[\mathrm{CL}]$ ) for functions whose domain is an abstract metric-measure space (see also $[\mathrm{R}]$ ). Compare $[\mathrm{S}],[\mathrm{DS}],[\mathrm{M} 1]$ and $[\mathrm{Ca}]$.

Acknowledgements. The author would like to thank the Research Council of Sharif University of Technology for support.

\section{References}

[AC] A. Alberico and A. Cianchi, Differentiability properties of Orlicz-Sobolev functions, Ark. Mat. 43 (2005), 1-28.

[BS] C. Bennett and R. Sharpley, Interpolation of Operators, Pure Appl. Math. 129, Academic Press, Boston, 1988.

[Ca] A. P. Calderón, On the differentiability of absolutely continuous functions, Riv. Mat. Univ. Parma 2 (1951), 203-213.

[Cc] C. P. Calderón, Lacunary differentiability of functions in $\mathbb{R}^{n}$, J. Approx. Theory 40 (1984), 148-154.

[CL] C. P. Calderón and J. Lewis, Maximal smoothing operators and some Orlicz classes, Studia Math. 57 (1976), 285-296.

[CRS] M. J. Carro, J. A. Raposo and J. Soria, Recent developments in the theory of Lorentz spaces and weighted inequalities, Mem. Amer. Math. Soc. 187 (2007), no. 877 .

[DS] R. A. DeVore and R. C. Sharpley, On the differentiability of functions in $\mathbb{R}^{n}$, Proc. Amer. Math. Soc. 91 (1984), 326-328. 
[GT] D. Gilbarg and N. S. Trudinger, Elliptic Partial Differential Equations of Second Order, Classics Math., Springer, Berlin, 2001.

[H] P. Hajłasz, Sobolev spaces on an arbitrary metric space, Potential Anal. 5 (1996), 403-415.

[HK] P. Hajłasz and P. Koskela, Sobolev met Poincaré, Mem. Amer. Math. Soc. 145 2000, no. 688 .

[He] J. Heinonen, Lectures on Analysis on Metric Spaces, Springer, New York, 2001.

[KKM] J. Kauhanen, P. Koskela and J. Malý, On functions with derivatives in a Lorentz space, Manuscripta Math. 100 (1999), 87-101.

[KK] V. Kokilashvili and M. Krbec, Weighted Inequalities in Lorentz and Orlicz Spaces, World Sci., River Edge, NJ, 1991.

[M1] J. Malý, Absolutely continuous functions of several variables, J. Math. Anal. Appl. 231 (1999), 492-508.

[M2] - Coarea properties of Sobolev functions, in: Function Spaces, Differential Operators and Nonlinear Analysis (Teistungen, 2001), Birkhäuser, Basel, 2003, 371381.

[MS] Y. Mizuta and T. Shimomura, Continuity and differentiability for weighted Sobolev spaces, Proc. Amer. Math. Soc. 130 (2002), 2985-2994.

[R] A. Ranjbar-Motlagh, Generalized Stepanov type theorem with applications over metric-measure spaces, Houston J. Math. 34 (2008), 623-635.

[S] E. M. Stein, Editor's note: the differentiability of functions in $\mathbb{R}^{n}$, Ann. of Math. (2) 113 (1981), 383-385.

[SW] E. M. Stein and G. Weiss, Introduction to Fourier Analysis on Euclidean Spaces, Princeton Math. Ser. 32, Princeton Univ. Press, Princeton, 1975.

Department of Mathematical Sciences

Sharif University of Technology

P.O. Box 11365-9415

Tehran, Iran

E-mail: ranjbarm@sharif.edu

Received June 6, 2007

Revised version June 18, 2008 